\title{
Excess Mortality Caused by Medical Injury
}

\author{
Linda N. Meurer, $M D, M P H^{1,2}$ \\ Hongyan Yang, $M S^{1}$ \\ Clare E. Guse, $M S^{1,2}$ \\ Carla Russo, $M D^{1}$ \\ Karen J. Brasel, MD, MPH',3 \\ Peter M. Layde, MD, MSc ${ }^{1,2}$ \\ 'Department of Family and Community \\ Medicine, Medical College of Wisconsin, \\ Milwaukee, Wisc \\ ${ }^{2}$ Injury Research Center, Medical College \\ of Wisconsin, Milwaukee, Wisc \\ ${ }^{3}$ Department of Surgery, Medical College \\ of Wisconsin, Milwaukee, Wisc
}

\begin{abstract}
PURPOSE We wanted to estimate excess risk of in-hospital mortality associated with medical injuries identified using an injury surveillance system, after controlling for risk of death resulting from comorbidities.

METHODS The Wisconsin Medical Injuries Prevention Program (WMIPP) screening criteria were used to identify medical injuries, defined as "any untoward harm associated with a therapeutic or diagnostic healthcare intervention," among discharge diagnoses for all 562,317 patients discharged from 134 acute care hospitals in Wisconsin in 2002. We then derived estimates for crude and adjusted relative risk of in-hospital mortality associated with the presence of a medical injury diagnosis. Logistic regression adjusted for baseline risk of mortality using a comorbidity index, age, sex, Diagnosis Related Groups, hospital characteristics, and clustering within hospital.
\end{abstract}

RESULTS There were 77,666 discharges that met WMIPP criteria for at least 1 medical injury (13.8\%). Crude risk ratios for death ranged from 1.27 to 2.4 for those with medical injuries within 1 of 4 categories: drugs/biologics; devices, implants, and grafts; procedures; and radiation. After adjustment, estimates of excess mortality decreased, and significance persisted only for injuries related to procedures (39\%; 95\% confidence interval [Cl], 28\%-52\%) and devices, implants, and grafts (16\%; 95\% Cl, 3\%-30\%).

CONCLUSIONS Estimates of excess mortality that do not account for baseline mortality risk may be exaggerated. Findings have implications for the care family physicians provide in the hospital and for the advice they give their patients who are concerned about the risks of hospitalization.

Ann Fam Med 2006;4:410-416. DOI: 10.1370/afm.553.

\section{INTRODUCTION}

$\mathrm{F}$ amily physicians provide ongoing medical care for a large propor$\checkmark$ tion of patients in the United States. According to the 2003 National Ambulatory Care Survey, 25\% of all physician office visits were to family physicians. ${ }^{1}$ Between $55 \%$ and $80 \%$ of family physicians care for their adult patients when admitted to the hospital, ${ }^{2}$ and all family physicians have some role in preoperative and follow-up care, care coordination, education, and counseling regarding the need for hospitalization. Further, $35 \%$ to $61 \%$ of adverse events identified in hospitalized patients occur before their hospitalization. ${ }^{3}$ As patient advocates and first contact with the health care system, family physicians play an important and underrecognized role in preventing and remediating injuries that occur during the course of health care, in and out of the hospital. Accordingly, awareness and understanding of the evidence in regard to medical injury are critical to providing informed, proactive care.

The 1999 Institute of Medicine report To Err is Human ${ }^{4}$ estimated that between 44,000 and 98,000 people die annually as a result of medical errors, placing health care injury among the top 10 causes of death, more than motor vehicle crashes, breast cancer, and acquired immunodeficiency syndrome. ${ }^{5}$ These frighteningly high numbers raised concern and awareness about patient safety and have led to advances in the measurement 
and prevention of adverse events in the clinic and the hospital. ${ }^{6,7}$ These estimates, obtained by extrapolating data from 2 large studies based on chart reviews of thousands of hospital records, ${ }^{8-10}$ led to some controversy. ${ }^{11,12}$ One criticism of the IOM estimates is that the original studies lacked control for baseline mortality risk or illness severity and that many patients would have died in the absence of an adverse event. ${ }^{11}$

Zhan and Miller ${ }^{13}$ advanced estimation of patient mortality related to safety using administrative hospital discharge data to study the frequency and outcomes of the Agency for Healthcare Research and Quality (AHRQ) Patient Safety Indicators (PSIs). ${ }^{14,15}$ PSIs are a specific set of 20 indicators designed to identify events reflecting a high probability of poorquality inpatient care during a specific hospitalization. Using a comorbidity index to account for baseline differences in mortality risk, Zhan and Miller found that PSIs involving infections and postoperative complications were associated with significant increases in mortality risk.

These approaches and others use voluntary incident reporting to identify errors and near misses and focus on identifying sources of error or poor-quality care to improve safety. Many medical injuries occur, however, in the course of appropriate care. ${ }^{8,16} \mathrm{An}$ injury control model that focuses on patient outcomes provides a complementary framework for studying patient safety problems. ${ }^{16}$

\section{Wisconsin Medical Injury Prevention Program Approach}

The Wisconsin Medical Injury Prevention Program (WMIPP) screening criteria identify medical injuries, defined as "any untoward harm associated with a therapeutic or diagnostic healthcare intervention,"17 in routinely collected hospital discharge data. The criteria are based on 688 specific ICD-9 CM (International Classification of Disease, 9 th revision - Clinical Modification $)^{18}$ diagnostic $\mathrm{N}$ codes and, where relevant, E codes, which attribute an external cause to injuries, poisonings, and adverse events. Medical injuries are classified into 4 broad categories: drugs and biologics; procedures; devices, implants, and grafts; and radiation. Using a medical record review as a reference standard, and after adjusting for oversampling of records with medical injury diagnoses, WMIPP determined the screening criteria had a sensitivity of $60 \%$ and a specificity of $97 \% .{ }^{17}$

We used the WMIPP screening criteria to identify medical injuries among patients discharged from Wisconsin hospitals and to estimate associated excess mortality, crudely and after adjustment for baseline mortality risk. We hypothesized that some of the excess mortality associated with medical injury would be accounted for by confounding differences between patients with and without a medical injury.

\section{METHODS}

Hospital discharge data were obtained from the Wisconsin Bureau of Health Information. The Bureau of Health Information collected, edited and publicly disseminated summary data for all patients discharged from nonfederal, acute care hospitals in Wisconsin based on the Health Care Finance Administration uniform billing report form. The data include ICD-9 diagnostic and $\mathrm{E}$ codes for up to 10 discharge diagnoses, up to 6 procedure codes, length of stay, hospital charges, disposition at discharge, and other patient demographic and hospital characteristics.

To identify and categorize occurrences of medical injury codes, we applied WMIPP screening criteria to discharge diagnoses for all patient discharges from the 134 general, acute care hospitals in Wisconsin in 2002, excluding the delivery discharge records for normal newborns. Our study methods were reviewed and approved by the Medical College of Wisconsin's Institutional Review Board.

Statistical analysis was performed using Stata software. ${ }^{19}$ Rates of medical injuries were calculated as the number of discharge records with the particular identified medical injury code divided by the total number of discharges. The crude mortality rate ratio (RR) and 95\% confidence interval (CI) were calculated using standard methods for categorical data for patients whose discharge records had at least 1 medical injury code compared with those whose records had no medical injury code.

We used logistic regression to estimate odds ratios for mortality associated with medical injury after adjusting for baseline risk of death with Zhan and Miller's comorbidity index. This comorbidity index is based upon Elixhauser's 30 comorbidity measures, which account for underlying, preexisting conditions not related to the main reason for hospitalization, but which increase the likelihood of a poor outcome. ${ }^{13,20}$ The comorbidity index was derived by first estimating the regression coefficients of each of the comorbidities on in-hospital mortality and then summarizing those comorbidities that were significantly and positively associated with excess mortality into an index with a possible range of scores from 0 to $33.81 .{ }^{13} \mathrm{We}$ also adjusted for age, sex, Diagnostic Related Group (as a fixed effect), hospital characteristics (ownership, trauma center level, transplant services offered, teaching/nonteaching, percentage of board-certified staff, and specificity of ICD-9 coding), and clustering 
within hospital (as patients within specific hospitals may share characteristics). The intraclass correlation for clustering within hospitals was 0.0043 with death as the outcome variable.

\section{RESULTS}

By applying the screening criteria to the 562,317 eligible discharges from acute care hospitals in Wisconsin during 2002, we identified 77,666 patients $(13.81 \%)$ with at least 1 medical injury diagnosis. Patients who sustained a medical injury were more likely to be older, have a higher comorbidity index, and experience a longer length of stay and greater number of procedures (Table 1). The average comorbidity index for Wisconsin patients was significantly lower than that reported by Zhan and Miller, who used the AHRQ Healthcare Cost and Utilization Project National Inpatient Sample database (1.12 vs 3.77; $P<.001)$. Further, a greater proportion of our patients $(70 \%)$ had a comorbidity index of 0.0 when compared with the national inpatient sample (52\%). Rates of medical injury and mortality increased with increasing comorbidity index (Figure 1).

Overall, deaths occurred among $3.14 \%$ of those who also experienced a medical injury and $2.13 \%$ of those who had no medical injury diagnosis upon discharge $(\mathrm{RR}=1.47 ; 95 \% \mathrm{CI}$, 1.42-1.54). Mortality risk was elevated for each of the medical injury categories, ranging from 1.27 for drug-associated injury to 2.41 for radiation-associated injury (Table 2).

These unadjusted relative risks do not take into account the differences between patients who had and who did not have a medical injury with respect to baseline risk of death caused by
Table 1. Characteristics of Patients With and Without Medical Injury

\begin{tabular}{lcc}
\hline Characteristics & $\begin{array}{c}\text { Without Medical Injury } \\
(\mathbf{n}=\mathbf{4 8 4 , 6 5 0 )}\end{array}$ & $\begin{array}{c}\text { With Medical Injury } \\
(\mathbf{n}=\mathbf{7 7 , 6 6 6 )}\end{array}$ \\
\hline Mean age, years ( \pm SD) & $53.3 \pm 24.7$ & $59.2 \pm 21.4$ \\
$0-15, \%$ & 6.1 & 3.9 \\
$16-44, \%$ & 32.0 & 20.4 \\
$45-64, \%$ & 22.0 & 27.4 \\
$>65, \%$ & 39.9 & 48.3 \\
Sex, female, \% & 60.2 & 54.8 \\
Mean length of stay, days ( \pm SD) & $5.0 \pm 5.6$ & $8.0 \pm 9.5$ \\
Comorbidity index, mean ( \pm SD) & $1.00 \pm 1.99$ & $1.84 \pm 2.61$ \\
Number of procedures, \% & & \\
0 & 40.2 & 30.9 \\
1 & 25.9 & 22.3 \\
2 & 15.3 & 14.9 \\
3 & 9.1 & 10.0 \\
4 & 4.2 & 6.3 \\
5 & 2.6 & 5.9 \\
$6+$ & 2.9 & 9.7 \\
Procedure type, \% & & \\
No procedure & 40.2 & 30.9 \\
Operative only & 7.1 & 5.7 \\
Nonoperative only & 41.3 & 20.5 \\
Both & 11.4 & \\
\hline All differences statistically significant at $P<.001$. & & \\
* Comorbidity index score, with possible range of scores $0.0-33.81 ;$ higher score indicates greater comorbidity. & \\
\hline
\end{tabular}

Figure 1. Injury and mortality rates by comorbidity index.

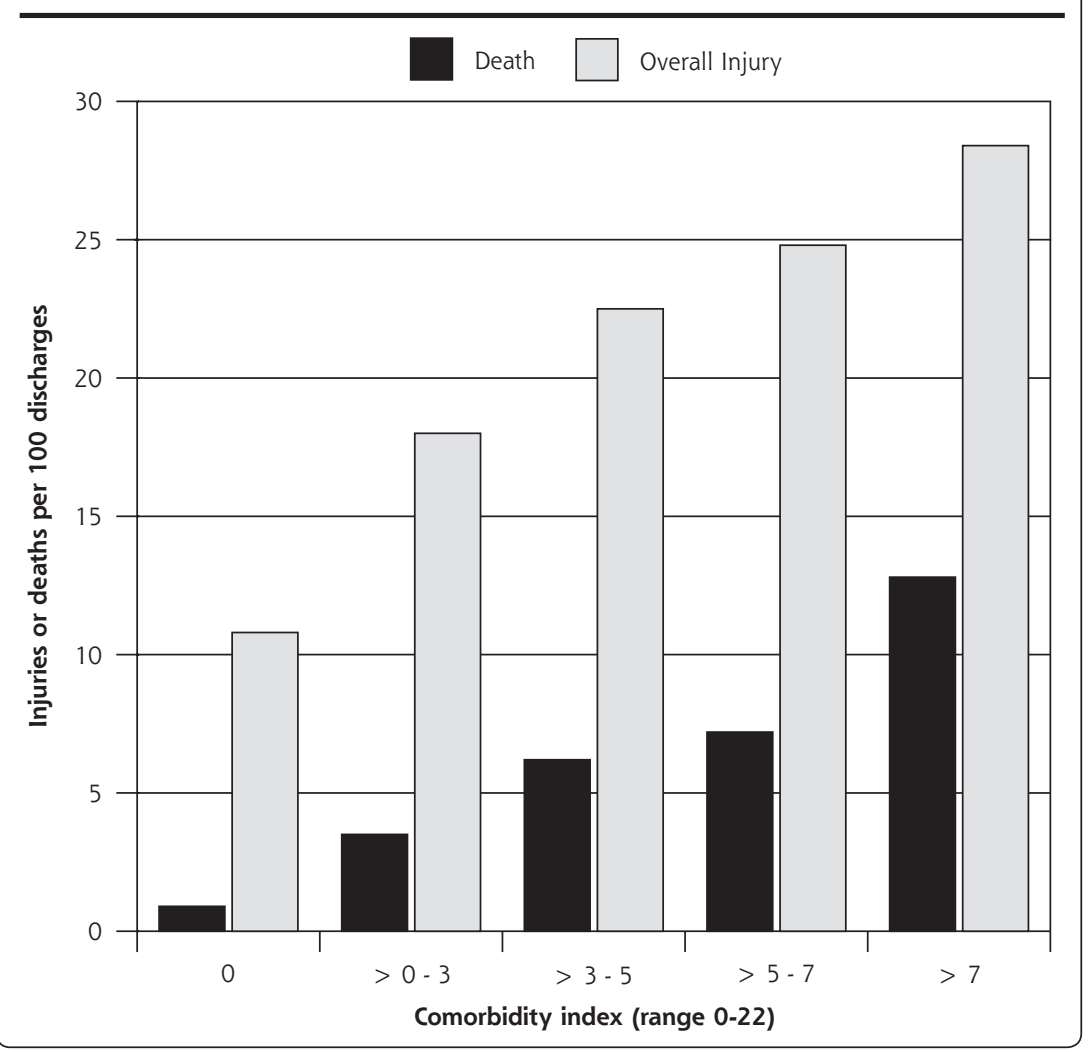




\begin{tabular}{|c|c|c|c|c|c|}
\hline Measure & $\begin{array}{l}\text { Drugs and } \\
\text { Biologics }\end{array}$ & $\begin{array}{l}\text { Devices, } \\
\text { Implants and } \\
\text { Grafts }\end{array}$ & Procedures & Radiation & Overall \\
\hline $\begin{array}{l}\text { Number of discharges with medical } \\
\text { injury code }\end{array}$ & 33,787 & 17,146 & 33,578 & 1,545 & $77,666^{*}$ \\
\hline Medical injury rate, \% & 6.01 & 3.05 & 5.97 & 0.27 & 13.81 \\
\hline $\begin{array}{l}\text { Crude mortality rate among those } \\
\text { with specific medical injury, \% }\end{array}$ & 2.84 & 3.52 & 3.54 & 5.44 & 3.14 \\
\hline $\begin{array}{l}\text { Crude mortality rate among those } \\
\text { without specific medical injury, \% }\end{array}$ & 2.23 & 2.23 & 2.19 & 2.26 & 2.13 \\
\hline Crude rate ratio (RR) & 1.27 & 1.58 & 1.62 & 2.41 & 1.47 \\
\hline $\begin{array}{l}\text { Adjusted odds ratio } \\
(95 \% \mathrm{Cl})\end{array}$ & $\begin{array}{c}0.73^{\ddagger} \\
(0.66-0.81)\end{array}$ & $\begin{array}{c}1.16^{\ddagger} \\
(1.03-1.30)\end{array}$ & $\begin{array}{c}1.39^{\ddagger} \\
(1.28-1.52)\end{array}$ & $\begin{array}{c}1.10 \\
(0.91-1.34)\end{array}$ & $\begin{array}{c}0.98 \\
(0.91-1.06)\end{array}$ \\
\hline $\begin{array}{l}\text { * Total is less than sum of categories as so } \\
\dagger \text { Adjusted for comorbidity index, age, sex } \\
\text { of board certified staff, transplant services } \\
\ddagger P<.05 \text {. }\end{array}$ & $\begin{array}{l}\text { records include } m \\
\text { lagnosis Related } \\
\text { rcentage of nons }\end{array}$ & $\begin{array}{l}\text { than } 1 \text { injury code. } \\
\text { p (as fixed effect), ho } \\
\text { ic coding), and cluste }\end{array}$ & $\begin{array}{l}\text { characteristics (o } \\
\text { within hospital. }\end{array}$ & , trauma level, & training, percen \\
\hline
\end{tabular}

comorbidities and other potential confounders. When these factors were accounted for using a logistic regression model, the excess risk of mortality associated with any medical injury disappeared (adjusted odds ratio = 0.98; 95\% CI, 0.91-1.06) (Table 2). Adjusted odds ratios of mortality for the categories of type of medical injury were all reduced compared with the corresponding unadjusted relative risks. Injuries related to procedures or to a device, implant, or graft were associated with increased adjusted odds of mortality of 39\% (95\% CI, $28 \%-52 \%)$ and $16 \%$ (95\% CI, 3-30\%), respectively. There was no increased mortality associated with radiation-related medical injury, and the finding of a drugrelated medical injury code was actually associated with a significantly lower mortality risk.

Closer examination of the WMIPP codes in these data showed that the most cases involved drug side effects, or "adverse effects of drugs, properly administered." Although common, medication side effects are often not life-threatening (eg, rash, nausea). Given the limited number of fields on the uniform billing report form, we hypothesized that patients who are more seriously ill would have multiple comorbidities and other diagnoses, and they would therefore be less likely than an otherwise healthy patient to have a minor drug side-effect listed as one of their diagnoses codes. We therefore examined the relationship among total number of diagnosis codes, mortality, and the coding of a medical injury. Indeed, whereas mortality increased with number of coded diagnoses, rates of recorded medication-related injury diagnoses decreased as the total number of recorded diagnoses rose above 8 (Figure 2). This trend was not apparent for the other categories (data shown for drug- and procedure-related injuries).

\section{DISCUSSION}

Ultimately, the goal of medical care is to reduce illness and save lives. Even so, the relationships among severity of illness, exposure to medical procedures, risk of medical injury, and mortality are complex (Figure 3 ). At the extreme, an increased baseline risk of death affects decisions to undertake heroic measures-lifesaving procedures that may carry greater risk of injury themselves. These complicated relationships present challenges to researchers and clinicians, as data about medical injury are difficult to obtain and interpret. Errors in care are generally underreported, and chart reviews are hindered by incomplete data, the bias of hindsight, and disagreement among external reviewers. ${ }^{21-23}$ Caplan et al $^{21}$ found that even with otherwise equal information, retrospective judgments of the appropriateness of care is significantly affected by the physician's knowledge of the severity of the outcome.

Using administrative data, we found that that $13.8 \%$ of patients in Wisconsin hospitals experienced a medical injury, and that sustaining a medical injury was associated with a crude increase in mortality risk of $48 \%$. When extrapolated to the 31.3 million hospital admissions (excluding normal newborns) in the United States in 2002, ${ }^{24}$ this risk corresponds to an estimated 45,600 deaths annually associated with medical injury diagnoses. Though we define medical injury by harm, rather than by error, this number is similar to previous estimates of adverse events that form the basis of the IOM report.

Both medical injury and in-hospital mortality risks were higher among older patients who had multiple comorbidities at admission. After controlling for the baseline mortality risk using Zhan and Miller's comorbidity index, our estimated excess risk of mortality associated with medical injury dropped to less than 


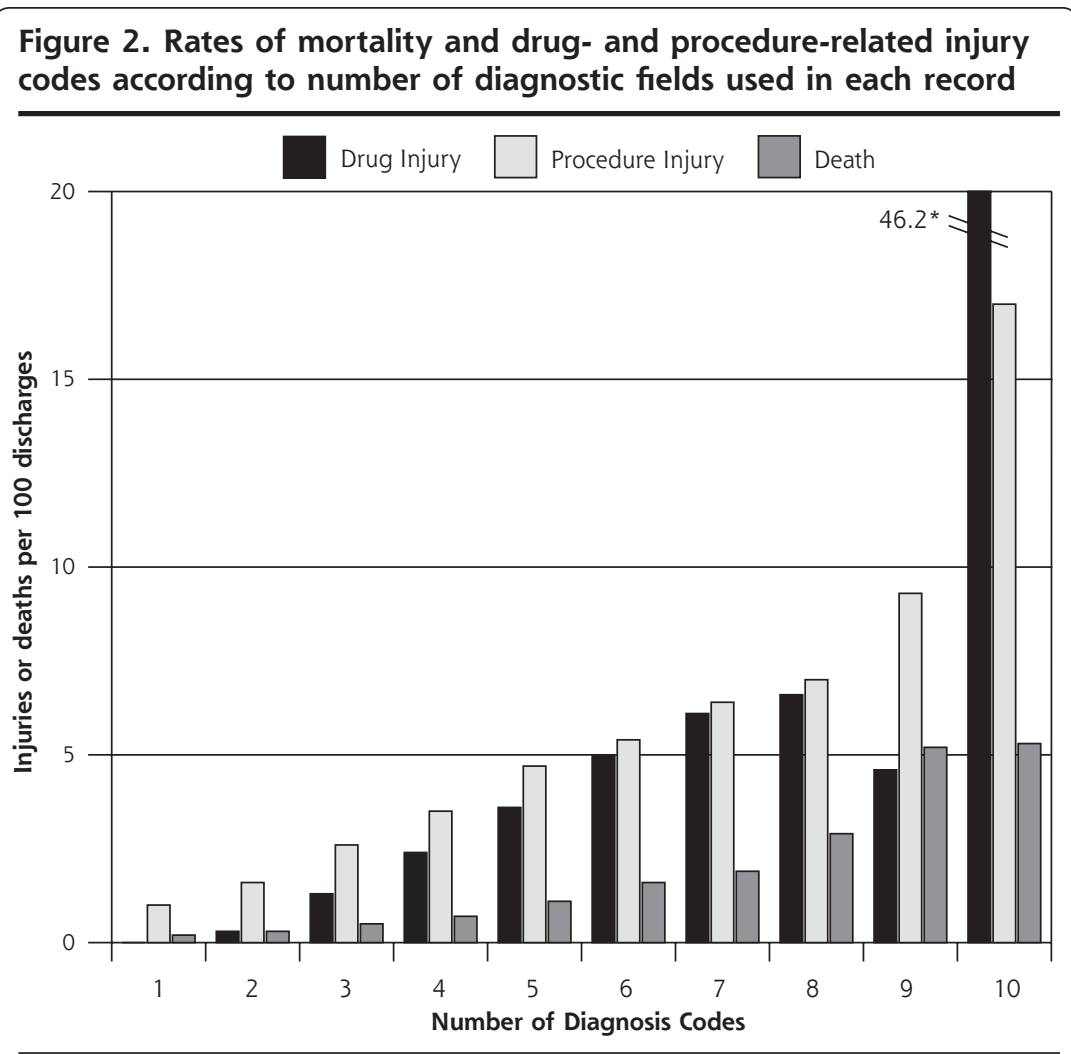

*Note: Diagnosis field \#10 is exclusively an E code (cause of injury) though E codes can also appear in other diagnosis fields. For this field, the average number of $E$ codes for drug injuries is 46.2 , exceeding the limits of this graph.

Figure 3. Interrelationships among illness severity, medical care, injury, and death.

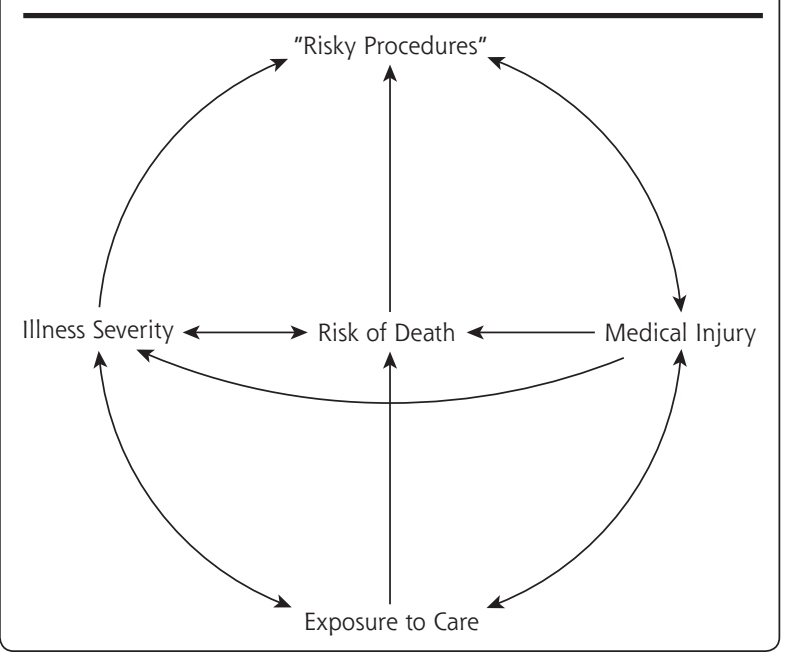

one half the unadjusted estimates. The excess mortality was primarily accounted for by procedure-related complications or injuries related to devices, implants, and grafts, a finding consistent with earlier work. ${ }^{13}$ Extrapolating these data nationally corresponds to roughly 1.95 million procedure-related injuries and 1 million device-related injuries, and assuming no overlap, an adjusted best estimate of 19,400 excess deaths.

WMIPP criteria are designed to identify medical injuries, or harm that occurs as a result of diagnostic or therapeutic health care interventions. Unlike chart reviews and adverse event reporting, these criteria do not identify cases of delayed diagnosis and therefore the delay or absence of intervention. WMIPP also uses a broad definition of injury to include all reported harms, not only those that result in permanent or severe disability. By using an injury control model, WMIPP criteria capture a different but overlapping subgroup of patients affected by patient safety problems, which complements other surveillance methods but makes direct comparison to earlier estimates based on chart review unwarranted. The substantial impact on estimates of excess mortality obtained by adjusting for baseline mortality risk suggests, however, that the number of deaths directly attributable to medical injury may not be as high as previous unadjusted estimates, and that future research in this area should include further development of methods for such adjustments.

With better understanding of the scope and consequences of medical injury, family physicians can take a proactive role in patient safety through facilitating hospitalization decisions, coordinating care and managing chronic illness, and responding promptly to injuries that appear or are carried into the posthospitalization period. Physicians must advise patients (especially those with multiple comorbidities) of the potential incidence and impact of medical injury, particularly if there are interventional and noninterventional approaches for treating a certain condition. More detailed examination of the characteristics of patients who suffer medical injury from specific procedures may help to assess risk for individual patients and guide treatment recommendations for patients at higher risk. In addition, by taking lessons from studies using hospital-based administrative data, we may be better equipped to design systems to monitor and detect medical injuries in the outpatient setting, thus adding to our armamentarium of patient safety tools. 
Administrative data are dependent on the quality and completeness of coding and reporting practices, and lack clinical detail. Even so, the breadth and wide availability of hospital discharge data provide an efficient means to identify trends, suggest associations, and generate hypotheses for further study of patient safety. Using a broad definition of harm, such as that used by WMIPP criteria, enables surveillance of the wide spectrum of medical injuries treated in hospitals, whether originating in the community or occurring during specific hospitalization. Such a system could complement error reporting and chart reviews in the ambulatory setting as well, but would require uniform coding practices and systems designed to recognize and document harm caused by medical interventions. Recent enhancements of the hospital discharge data to include admission diagnoses will enable researchers to better delineate the source of injuries in the future, and facilitate prospective studies and the study of injuries related to outpatient care.

This study is limited by accuracy of administrative coding practices and the completeness of ascertainment of medical injuries using ICD E code and $\mathrm{N}$ codes. In Wisconsin, external cause of injury (E) coding is required for relevant $\mathrm{N}$ codes ( $\mathrm{N}$ 800-996); relevant records that do not contain an $\mathrm{E}$ code are returned to the submitting hospital for completion. For motor vehicle crashes, external cause of injury (E) coding is very complete as a result, ${ }^{25}$ but $\mathrm{E}$ coding rates for medical inujuries have not been studied. WMIPP criteria rely on information obtained or coded in the chart and therefore are likely represent an undercounting of harmful events, particularly among patients with multiple comorbidities. For drug-related injuries, patients with multiple diagnoses were less likely to have an injury code recorded, which may explain the finding that drug-related medical injuries were actually associated with a decreased adjusted risk of mortality. Relatively minor "adverse effects to drugs properly administered," such as nausea or dermatitis, may be coded more often for patients with otherwise uncomplicated hospital stays. The same may also be true to a lesser extent for other categories, leading to a possible underestimate of the risk of death attributable to an injury category. The complexities related to coding given the large numbers of diagnoses warrants further examination.

Even so, death is not the only result of medical injury worthy of attention. Regardless of how adverse events in health care are defined or identified, their numbers remain consistently high. In 2002, nearly 78,000 patients in Wisconsin alone experienced a medical injury, defined as harm, regardless of whether that harm resulted in death, major disability, increased costs or length of stay, or more immeasurable but real costs to the individual, such as pain, discomfort, or fear. Whereas attention to deaths and disabling injuries is critically important, limiting the focus to only the most severe outcomes may skew both the health care community's and the public's perception of the extent and impact of injuries related to health care.

To read or post commentaries in response to this article, see it online at http://www.annfammed.org/cgi/content/full/4/5/410.

Key words: Hospital mortality; safety, medical device; medication errors; postoperative complications; adverse effects; iatrogenic disease; comorbidity; medical errors; quality of health care; research methods

Submitted August 23, 2005; submitted, revised, January 16, 2006; accepted January 30, 2006.

A version of this report was presented as a distinguished paper at the North American Primary Care Research Group Annual Meeting in Orlando, Fla, October 2004.

Funding support: This project was supported in part by the Agency for Health Research and Quality (U18 HS11893) and by the Centers for Disease Control and Prevention (R49/CCR519614).

Acknowledgments: We would like to thank Chris McLaughlin for her skilled and thoughtful editorial assistance.

\section{References}

1. Woodwell DA, Cherry DK. National Ambulatory Medical Care Survey: 2002 summary. Adv Data. 2004:1-44.

2. American Academy of Family Physicians. 2005 Facts about Family Medicine. Practice Profile I Survey. May, 2005. Available at: http:// www.aafp.org/x530.xml\#x729. Accessed: 29 November 2005.

3. Forster AJ, Asmis TR, Clark HD, et al. Ottawa Hospital Patient Safety Study: incidence and timing of adverse events in patients admitted to a Canadian teaching hospital. CMAJ. 2004;170:1235-1240.

4. Institute of Medicine. Committee on Quality of Health Care in America. To Err is Human: Building a Safer Health System. Washington, DC: National Academy Press; 2000.

5. Martin JA, Smith BL, Mathews TJ, Ventura SJ. Births and deaths: preliminary data for 1998. Natl Vital Stat Rep. 1999;47:1-45.

6. Dovey SM, Meyers DS, Phillips RL, Jr., et al. A preliminary taxonomy of medical errors in family practice. Qual Saf Health Care. 2002;11:233-238

7. Elder NC, Vonder Meulen M, Cassedy A. The identification of medical errors by family physicians during outpatient visits. Ann Fam Med. 2004;2:125-129.

8. Brennan TA, Leape LL, Laird NM, et al. Incidence of adverse events and negligence in hospitalized patients. Results of the Harvard Medical Practice Study I. N Engl J Med. 1991;324:370-376.

9. Leape LL, Brennan TA, Laird N, et al. The nature of adverse events in hospitalized patients. Results of the Harvard Medical Practice Study II. N Engl J Med. 1991;324:377-384.

10. Thomas EJ, Studdert DM, Burstin HR, et al. Incidence and types of adverse events and negligent care in Utah and Colorado. Med Care. 2000;38:261-271.

11. McDonald CJ, Weiner M, Hui SL. Deaths due to medical errors are exaggerated in Institute of Medicine report. JAMA. 2000;284:93-95. 
12. Leape LL. Institute of Medicine medical error figures are not exaggerated. JAMA. 2000;284:95-97.

13. Zhan C, Miller MR. Excess length of stay, charges, and mortality attributable to medical injuries during hospitalization. JAMA. 2003;290:1868-1874.

14. Miller MR, Elixhauser A, Zhan C, Meyer GS. Patient safety indicators: using administrative data to identify potential patient safety concerns. Health Serv Res. 2001;36:110-132.

15. Romano PS, Geppert J], Davies S, et al. A national profile of patient safety in U.S. hospitals. Health Aff (Millwood). 2003;22:154-166.

16. Layde PM, Cortes LM, Teret SP, et al. Patient safety efforts should focus on medical injuries. JAMA. 2002;287:1993-1997.

17. Layde PM, Meurer LM, Guse CE, et al. Medical injury identification using hospital discharge data. In: Advances in Patient Safety: From Research to Implementation. Rockville, MD: Agency for Healthcare Research and Quality; 2005. AHRQ Publication Nos. 050021 (1-4). Vol. 2;119-132. Available at: http://www.ahrq.gov/qual/advances/.

18. US Department of Health and Human Services. International Classification of Diseases 9th Revision - Clinical Modification. 5th ed. Hyattsville, Md: Centers for Disease Control and Prevention; 1999.
19. Stata Statistical Software. Release 8.0. College Station, Tex: StataCorp; 2003.

20. Elixhauser A, Steiner C, Harris DR, Coffey RM. Comorbidity measures for use with administrative data. Med Care. 1998;36:8-27.

21. Caplan RA, Posner KL, Cheney FW. Effect of outcome on physician judgments of appropriateness of care. JAMA. 1991;265:1957-1960.

22. Localio AR, Weaver SL, Landis JR, et al. Identifying adverse events caused by medical care: degree of physician agreement in a retrospective chart review. Ann Intern Med. 1996;125:457-464.

23. Hayward RA, Hofer TP. Estimating hospital deaths due to medical errors: preventability is in the eye of the reviewer. JAMA. 2001;286:415-420

24. Kozak LJ, Owings MF, Hall MJ. National Hospital Discharge Survey: 2002 annual summary with detailed diagnosis and procedure data. Vital Health Stat 13. 2005:1-199.

25. Tavris DR, Kuhn EM, Layde PM. Hospitalizations for vehicle associated injuries in Wisconsin. WMJ. 1999;98:34-39. 